\title{
IoT task management system using control board
}

\author{
Changsu Kim, Youngkuk Kim, Hoekyung Jung \\ Department of Computer Engineering, Paichai University, 155-40 Baejae-ro, Seo-gu, Daejeon, South Korea
}

\begin{tabular}{l}
\hline \hline Article Info \\
\hline Article history: \\
Received Aug 21, 2018 \\
Revised Nov 5, 2018 \\
Accepted Nov 19, 2018 \\
\hline Keywords: \\
Control board \\
Data analysis \\
IoT \\
Remote control \\
Sensor \\
\hline
\end{tabular}

\begin{abstract}
Recently, Internet of Things (IoT) has been providing services that increase convenience for users by connecting objects to a network. As a result, the number of users and smart devices utilizing IoT is also increasing. So, the existing system has a problem that when a variety of devices are added, a bottleneck or an overload of the server occurs, because of structure of the system. In this paper, we propose IoT task management system using control board to solve these problems. The server performs only simple communication and analysis, and the management of tasks such as remote control is carried out using the control board. Also, it analyzes the user's remote control command and recommends to users the most used equipment. Through this, it is possible to reduce the data throughput and traffic of the server and to provide the service with increased convenience and accuracy of operation.
\end{abstract}

Copyright $@ 2019$ Institute of Advanced Engineering and Science. All rights reserved.

\section{Corresponding Author:}

Hoekyung Jung,

Department of Computer Engineering,

Paichai University,

155-40 Baejae-ro, Seo-gu, Daejeon, South Korea.

Email: hkjung@pcu.ac.kr

\section{INTRODUCTION}

Recently, IoT can be used regardless of location and time by connecting objects to network [1-4]. When applied in the industrial field, IoT can be applied to fine or repetitive tasks that are difficult for people to control, so that they can work quickly and efficiently. When it is used in the home, it provides users with convenient service such as remote control and monitoring [5-7]. Existing systems use centralized processing model to process tasks and data, which can lead to server overload if the number of devices connected increases $[8,9]$. In addition it provides a consistent service without considering the characteristics of users. Therefore, there is a problem that the convenience of the user and the accuracy of the operation are low $[3,5],[10,11,12]$.

In this paper, in order to solve these problems, we propose an IoT task control system using the control board. In order to reduce the burden on the server, the control board is used as an intermediate manager to process the sensor data and manage the work. The function of the control board is to receive sensor data and device information from Arduino and save each data into the database through 1:1 communication with the server. It also controls the device by transmitting user's remote control and reservation command to Arduino.

And, we analyze the recommendation device by time to provide the service considering the characteristics of users. When the user executes the remote control, the remote control command loaded to the database, and analysis is started by transmitting the analysis request to the server through the socket communication. The analysis utilizes the remote control commands stored in the database to determine which device the user has used at a particular time.

The result of the analysis is that the most-used device is transmitted to the Android application and is recommended to the user. This methodology will reduce the data throughput and traffic of the server and minimize the overload. 


\section{SYSTEM DESIGN}

The system uses the control board to process the device operation commands and sensor data. This methodology reduces the server's data throughput and traffic, thereby reducing overhead. In addition, user's remote control command is collected to derive the most used device by time and application recommends user what most used the device. This methodology can reduce the burden on the server and provide customized services to users. Figure 1 shows the configuration of the proposed system.

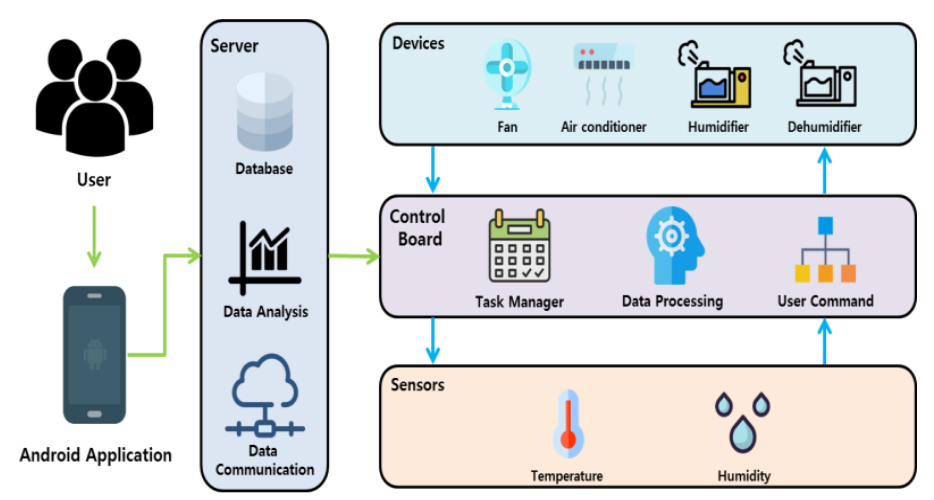

Figure 1. Block diagram for task management system

When the user executes the remote control or reservation function in the application, the command data is transmitted to the server. The remote control commands and the reservation data loaded to the database table and the command is transmitted to the control board. And, the control board transfers the received command directly to the device and processes it. Figure 2 shows the structure of the system.

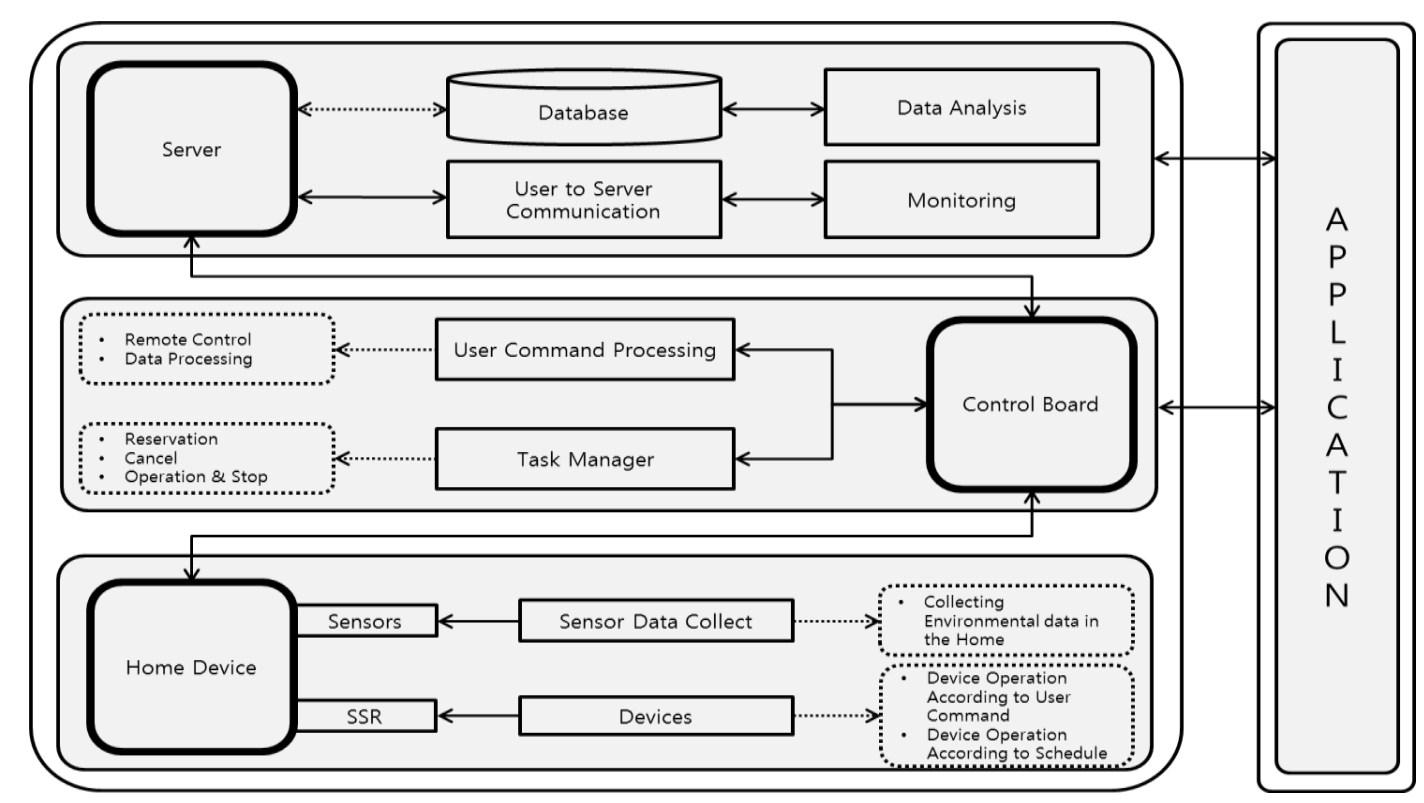

Figure 2. Architecture of task management system

The home device module consists of the devices and the sensors attached to the devices. Through the sensor, the environment data of the home is collected and transmitted to the server. The server loads the received sensor data into the database. Also, when the user executes the remote control and reservation function by utilizing the application, the server loads the corresponding command into the database and transmits the command to the control board. Analysis of the most used devices by time is based on user's remote control command. In this way, the user derives the device that used the most remote control 
at a certain time. The operating device is recommended to the user through the application and receives the selection value of the user. Figure 3 is a flow chart of the most-used device analysis algorithm by time.

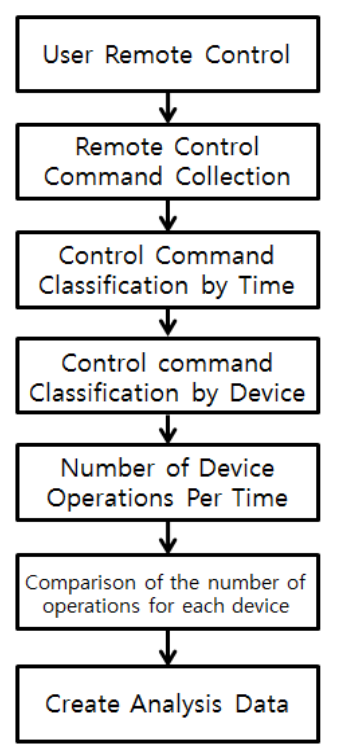

Figure 3. Analyze the most used devices by time flow

When the user executes the remote control using the application, the algorithm starts. The user's remote control command is collected, loaded to the database table, and utilized as analysis data. The loaded data is classified by time and reclassified for each device to derive the number of operation of the specific device at the relevant time. The number of operations per device is compared with each other to derive the most used device as a result at a specific time. The results of the analysis are transmitted to the application and the operation of the device is recommended to the user.

\section{SYSTEM IMPLEMENTATION}

The system utilizes the application to provide remote control, device operation scheduling, and monitoring service. Also, using the application, the user can control the device and obtain environmental information by checking the data of the current sensors. When the user utilizes the remote control of application, the operation and stop of each device can be controlled by using the ON and OFF buttons of the corresponding device. In addition, when the reservation function is utilized, a specific device can be operated and stopped at a desired time. Figure 4 shows the monitoring and remote control pages.

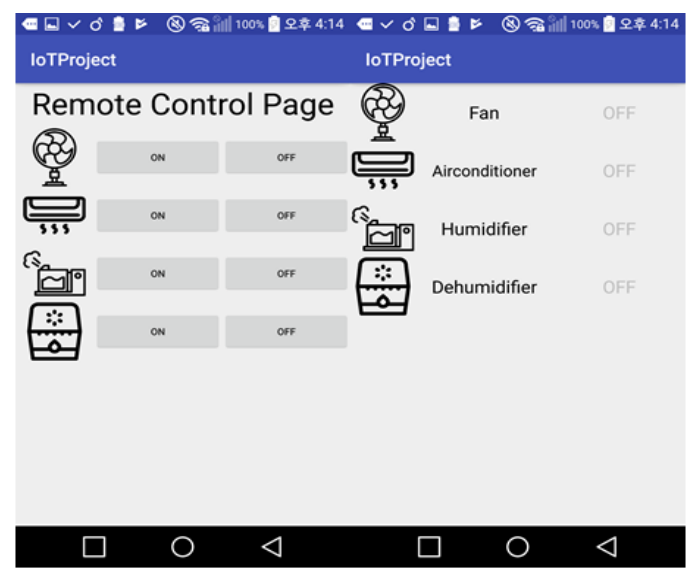

Figure 4. Remote control page and monitoring page 
The user can change the state of the device through the application. When using the remote control of application, it is possible to access the device and control the operation without being limited by the time and place. When remote control is performed, the change status value of the corresponding device is transmitted to the server and loaded into the database. The monitoring service collects the status of the device and sends it to the control board. The control board is transmitted to the server and transmitted to the application so that the status of the current device can be monitored.

Figure 5 shows the application recommends user that the devices. When the user starts the remote control through the application, it derives the most used device by time based on the data stored in the server. And, after the analysis result is transmitted to the application, the system provides customized service. Figure 6 shows the configuration of the overall system prototype.

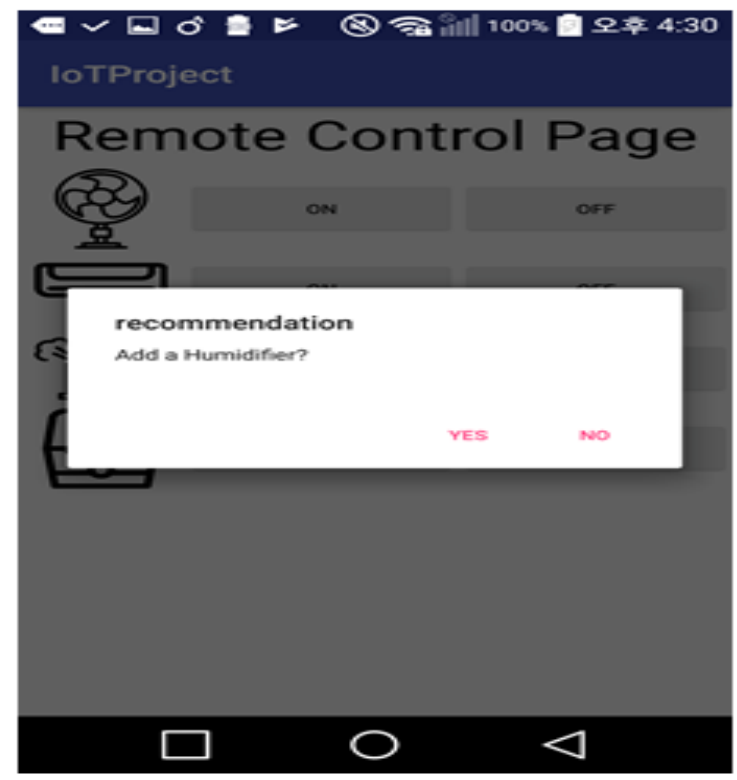

Figure 5. Device recommendation page

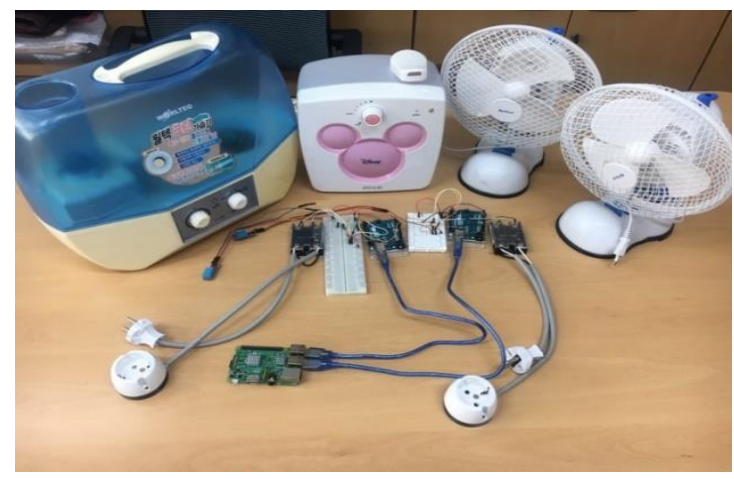

Figure 6. Prototype environment

In this system, a temperature sensor and a humidity sensor are attached to Arduino to measure the environment inside the home, and the environment is configured using Raspberry Pi as a control board. In addition, the operation of the device is carried out by an electric fan, an air conditioner, a humidifier, and a dehumidifier. When the user performs remote control, the user's command is sent to the server, and the server loads the remote control command to the database table and sends it to the control board. The control board receives the command of the user, determines the operation of the device, and transmits the command to the device to operate the device. For the experiment, the air conditioner was replaced with a fan, and the dehumidifier was replaced with a humidifier. 


\section{REVIEW}

In this study, to solve the problems of the existing IoT system, this study implemented the IoT system which can reduce the throughput and communication amount of the server by using the control board, analyze the characteristics of users and provide customized services. When the user starts the remote control by utilizing the application, the remote control command is transmitted to the server. The server loads the received remote control command into the database and uses it as analysis data and transmits the remote control command to the control board. The control board receives the command and determines the operation of the device and controls the device. Table 1 shows the dataset for the experiment.

Table 1. Experimental Dataset

\begin{tabular}{cl}
\hline System Type & \multicolumn{1}{c}{ Description } \\
\hline Existing & Temperature $-26^{\circ} \mathrm{C}$ \\
System & Humidity $-23 \%$ \\
& Data Processing - Server \\
& Task Management - Server \\
& Data Analysis - Server \\
Proposed & Temperature $-26^{\circ} \mathrm{C}$ \\
System & Humidity $-23 \%$ \\
& Data Processing - Control Board \\
& Task Management - Control Board \\
& Data Analysis - Server \\
\hline
\end{tabular}

The data set used in the experiment has the same temperature and humidity of the existing system and the proposed system. This is to confirm the difference between data throughput and traffic in the way that the server handles data processing and Task management and how the control board processes data. Figure 7 compares the server data throughput of the proposed system with the existing system.

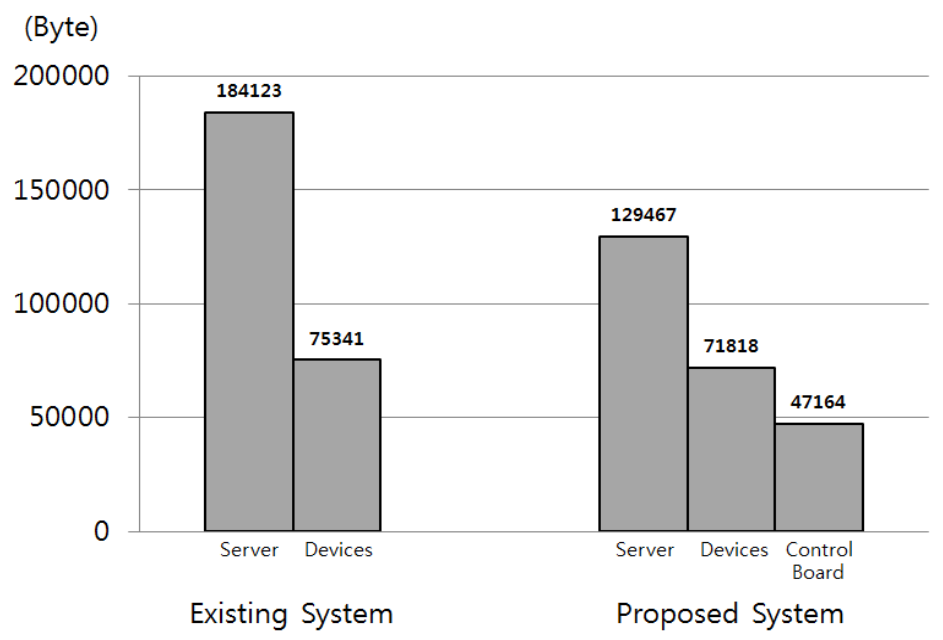

Figure 7. Server data throughput graph

Since the existing system uses the server and two devices to process the system, the server collects and processes all the data, and then proceeds to transmit the operation command of the device. Proposed system adds a control board and performs work management such as device operation discrimination, so that the data throughput of the server can be confirmed to be low. Figure 8 shows the data traffic of the existing system. 


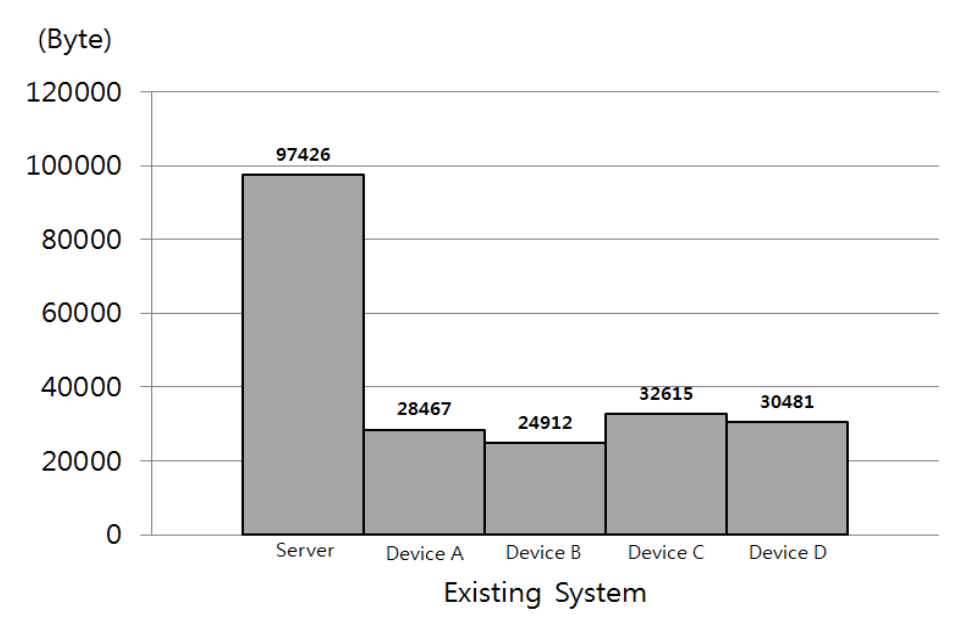

Figure 8. Existing system server data traffic graph

In the existing system, since the commands of the task are processed by the server, the workload of the server is higher than that of the device. Figure 9 shows the server traffic of the proposed system.

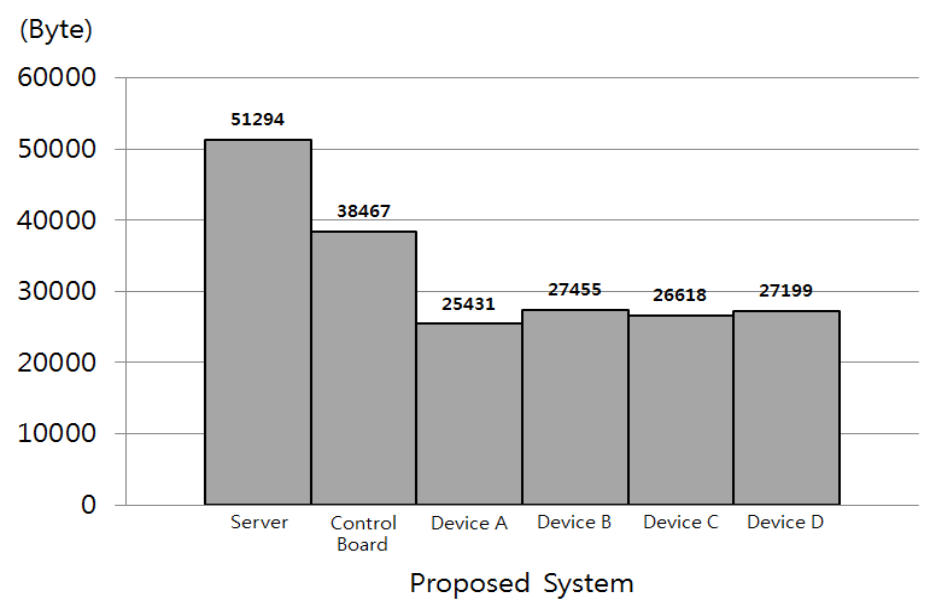

Figure 9. Proposed system server data traffic graph

\section{CONCLUSION}

In this paper, we proposed a task control system that provides services by analyzing characteristics of users by reducing server throughput and communication volume using control board. The system uses the application to send remote control commands to the server when the user starts remote control. The server loads the remote control command received from the application to the database table and transmits the remote control command to the control board to operate the devices. In addition, the remote control command of the user stored in the database is used as analysis data to analyze the most used devices by time. This reduces server traffic and data throughput and can provide services considering user characteristics. Future research is needed to increase the resource management efficiency of the control board, not the server.

\section{ACKNOWLEDGEMENTS}

This research was supported by The Leading Human Resource Training Program of Regional Neo industry through the National Research Foundation of Korea(NRF) funded by the Ministry of Science, ICT and future Planning(No. 2016H1D5A1911091). This work was supported by the research grant of Pai Chai University in 2018. 


\section{REFERENCES}

[1] J. A. Jeon, N. S. Kim, J. G. Go, T. J. Park, H. Y. Kang, C. S. Pyo. IoT Devices Product and Technology Trends. The Journal of The Korean Institute of Communication Sciences. 2014; 31(4): 44-52.

[2] S. Husain, A. Prasad, A. Kunz, A. Papageorgiou, and J. S. Song. Recent Trends in Standards Related to the Internet of Things and Machine-to-Machine Communications. Journal of Information and Communication Convergence Engineering. 2014; 12(4): 228-236.

[3] X. Bing. Key Internet of Things Technology and Application Research. TELKOMNIKA Indonesian Journal of Electrical Engineering. 2014; 12(7): 5599-5602.

[4] W. Yonghui. RFID SpatioTemporal Data Management. TELKOMNIKA Indonesian Journal of Electrical Engineering. 2013; 11(3): 1348-1354.

[5] L. Juntao. Research of IOTs Complex Event for Supply Chain Application. TELKOMNIKA Indonesian Journal of Electrical Engineering. 2013; 11(8): 4296-4305.

[6] C.S. Kim, H. K. Kim, H. K. Jung. IoT Distributed Processing System Based on Resource Allocation Algorithm. International Journal of Applied Engineering Research. 2017; 12(24): 15050-115054.

[7] S. Distefano, G. Merlino, A. Puliafito. A Utility Paradigm for IoT: The Sensing Cloud. Pervasive and mobile computing. 2015; 20: 127-114.

[8] Z. Xuezhi, S. K. Garg, P. Strazdins, P. P. Jayaraman, D. Georgakopoulos, R. Ranjan. IOTSim: A Simulator for Analysing IoT Applications. Journal of Systems Architecture. 2017; 72: 93-107.

[9] S. Trilles, A. Calia, O. Belmonte, J. T. Sospedra, R. Montoliu, J. Huerta. Deployment of an Open Sensorized Platform in a Smart City Context. Future Generation Computer Systems. 2017; 76: 221-233.

[10] T. H. Park, H. J. Seo, B. J. Bae, H. W. Kim. Secure Message Transmission Against Remote Control System. Journal of Information and Communication Convergence Engineering. 2016; 14(4): 233-239.

[11] K. Cao, H.J. Lee, H.K. Jung, Task Management System According to Changes in the Situation Based on IoT, Journal of Information Processing System. 2017; 13(6):1459-1466.

[12] J. H. Lee, S. Y. Cho, S. Park. Access Control for Arduino based Smart Home Using Web Interface. Korea Information Science Society. 2014; 1: 297-299. 